\title{
Can Additive Manufacturing Breathe New Life Into Aging Assets?
}

\author{
Stephen Fitzpatrick, Machining Team Lead, University of Strathclyde Advanced Forming Research Centre
}

Additive layer manufacturing technology has gained prominence in the aerospace sector and is being used for the rapid production of prototype parts. Its credibility has quite literally "taken off" and is now used to build inflight production components for aircraft. Its applications are being adopted in other sectors such as medical and automotive as a potentially faster and more economical alternative to traditional manufacturing methods for certain applications. Though it offers huge potential to the oil and gas arena, its uptake so far has been limited. The industry's risk-averse culture, lack of infrastructure, and stringent standards have been cited by leaders as barriers to adoption.

Yet, as experienced in other industries, the technical and economic benefits far outweigh the obstacles. The oil and gas industry's growing focus on operational efficiency in today's lowprice climate is slowly driving change and a realization that the challenges are not insurmountable.

Two main groups of technologies can be of benefit to the oil and gas industry: powder bed fusion and direct energy deposition. Both use a diverse range of metals in wire and powder form, which can be fused together using lasers, electron beams, and electric arcs.

\section{Asset Life Extension}

For aging oil and gas assets, replacement components may be difficult to source, require a long lead time to manufacture, and incur significant expense to produce. But the direct energy deposition additive technique called laser metal deposition could be the answer to remanufacture difficult-to-weld oil and gas components, such as those made of corrosion-resistant steels and heatresistant super alloys. Currently, no remanufacturing procedures exist for some high-value components, but by applying additive material to a specific area that is worn, you can extend the life of a component and actually increase its functional performance. This could play a key role in enabling oil and gas operators to reinstate their existing old equipment, reduce lead time, allow options to extend maintenance schedule cycles, and increase functional performance.

Laser metal deposition is a powder additive manufacturing process in which powders are conveyed through a nozzle and a laser is used to melt the layer of powder into a desired shape. This process offers many benefits for the oil and gas sector and, in particular, for remanufacturing applications. It has very fast cooling rates that create a very fine microstructure. There is also very low dilution and heat-affected

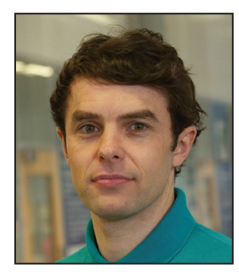

Stephen Fitzpatrick leads the AFRC's Machining and Additive Manufacturing Team in Glasgow, Scotland. Stephen also has extensive experience in the aerospace sector, where he had full engineering responsibility in the manufacturing of complex gas turbine aerospace components. Fitzpatrick is a chartered engineer of the Institute for Mechanical Engineers. He received an MSc degree in materials and manufacturing management from Sheffield Hallam University.

zone into the substrate material, meaning that thinner clad layers can be applied while still ensuring that the clad composition is achieved. A secondary benefit to lower dilution and a small heat-affected zone is that there will be shallower residual stress profiles, meaning very little distortion of components. This process can be applied very accurately, and fine features can be achieved with minimal post-process machining. Alloy powders can also be mixed together to create new alloys that can be functionally graded to obtain tailored properties.

Though this technology has been around for several years, its adoption has been slow, which may be due to a lack of control and insight into the process. With growing research, advanced optics and the availability of highpowered lasers now achieving the correct parameter windows, this additive process is realizing its potential. This is particularly relevant for remanufacturing applications where old assets can be reinstated into service to last beyond original design life. In some instances, there is an opportunity to avoid unnecessary scrapping and increase performance to that greater than the original component.

The University of Strathclyde Advanced Forming Research Centre (AFRC) is currently investigating deploying laser metal deposition for remanufacturing of aging assets, such as shafts, valves, and pumps, and as an alternative to traditional cladding of valves. A development process is initially undertaken so that porosity is mitigated and the correct parameter windows are identified to provide a quality output. This can then be tested and validated. 
There are other direct energy deposition technologies that could also have a significant impact on the industry, such as wire arc additive manufacturing to start building complete parts. This method allows you to build a component from nothing to a near-netshape state.

\section{Potential of Offshore 3D Printing}

Additive layer manufacturing, a form of 3D printing, also offers significant opportunities. The use of powder bed fusion additive processes to manufacture spares, for instance, is likely to increase, helping to save costs from not having to carry inventory. Other benefits include being able to rapidly produce complex prototype geometries and the consolidation of various parts into a single manufactured component bespoke to requirements, that may be difficult, if not impossible, to make using standard manufacturing techniques.

As manufacturing need not be constrained to a complex fabrication facility, further reaching benefits for the oil and gas industry also include reduced supply chain as well as the future potential to produce parts quickly, as and when required, on an offshore asset, which could significantly reduce the risk and costs associated with downtime.

Additive manufacturing and composite material systems will undoubtedly continue to replace many of the traditional uses of formed metallic parts and materials in the oil and gas industry. However, there is still an important set of conditions when forged and formed components are unquestionably the right answer-both economically and technically - to a particular design or manufacture challenge. Research carried out by the AFRC has shown that additive manufacturing can offer opportunities to improve existing routes of material production or even to replace some of them. However, further research is needed to fully understand the influence of different process parameters on microstructure and properties of the final product. JPT

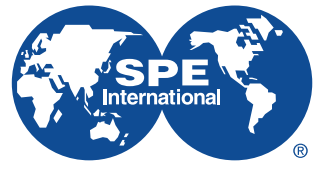

\section{SPE International}

\section{Conference and Exhibition on}

\section{Health, Safety, Security, Environment, and Social Responsibility}

16-18 April 2018

Jumeirah at Etihad Towers

Abu Dhabi, United Arab Emirates

\section{Register Today!}

Book your place today and save with the early bird fee.

\section{www.spe.org/go/reg4hse}
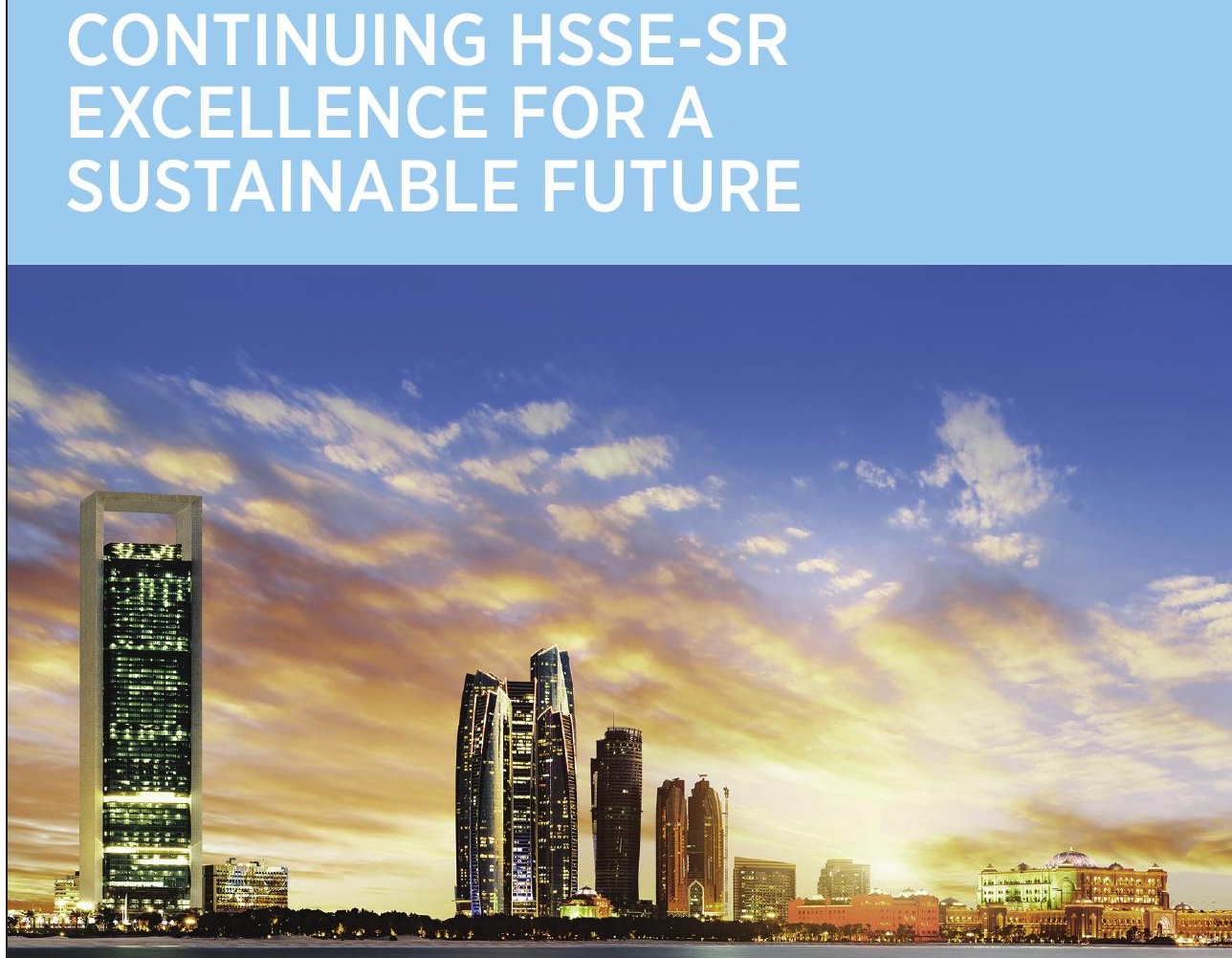

PRINCIPAL SPONSORS

PLATINUM SPONSORS DELEGATE BAGS SPONSOR .

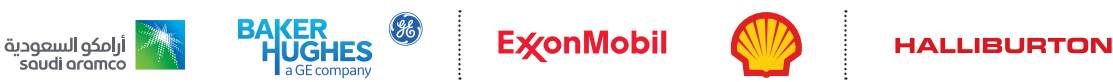

